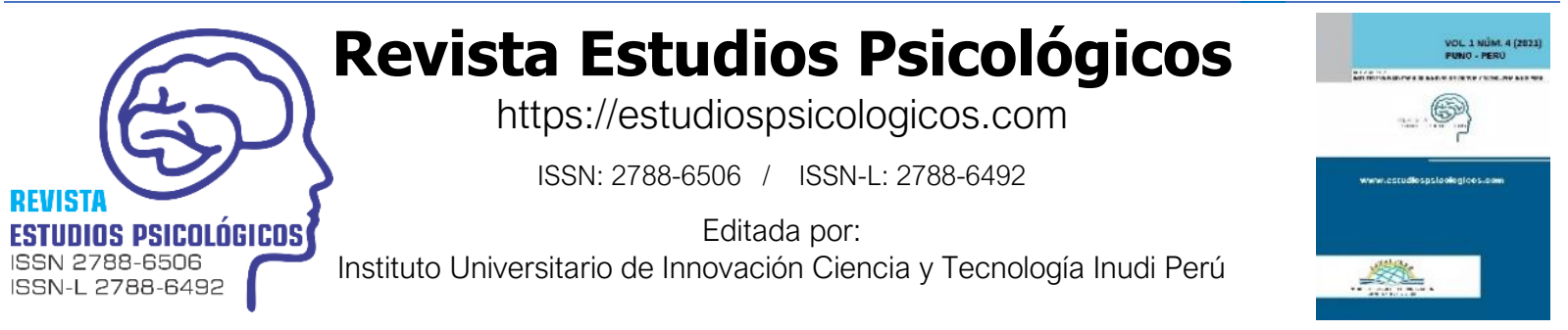

ARTÍCULO DE REVISIÓN

\title{
Activación conductual para el tratamiento de la depresión en pacientes oncológicos
}

\author{
Behavioral activation for the treatment of depression in cancer patients
}

Ativação comportamental para o tratamento da depressão em pacientes com câncer

\author{
Karina Pérez-Cárdenas ${ }^{1}$ \\ Universidad Católica de Cuenca, Cuenca - Azuay, Ecuador \\ (D) https://orcid.org/0000-0001-8397-6911
}

D0I: https://doi.org/10.35622/j.rep.2022.01.007

Recibido 24/09/2021/ Aceptado 10/01/2022
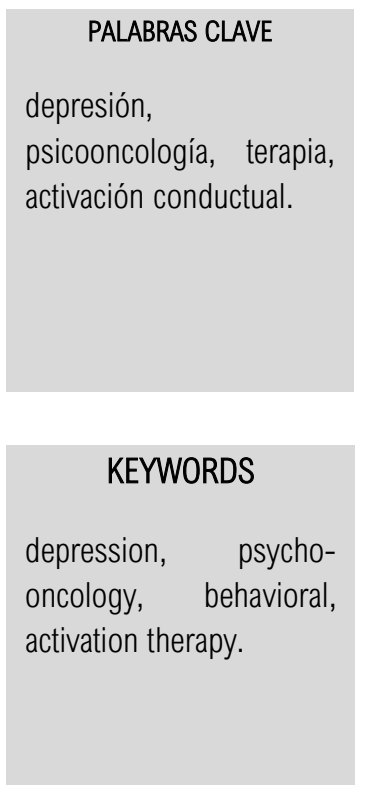

PALAVRAS-CHAVE
$\begin{aligned} & \text { depressão, } \\ & \text { oncologia, } \\ & \text { ativação comportamental. }\end{aligned}$

RESUMEN. La depresión en pacientes oncológicos es considerada como un problema psicológico prevalente, ya que los síntomas deprimentes se incrementan cuando se señala como una enfermedad crónica, en algunos casos se exceptúan actividades convirtiéndose en un obstáculo para el paciente en su desempeño de forma integral. Existen diversas técnicas psicoterapéuticas como coadyuvantes del tratamiento farmacológico para el control de la depresión, por lo tanto, este estudio pretende analizar la eficacia de terapéutica activación conductual en pacientes oncológicos con problemas de depresión. Se empleó un enfoque cualitativo en base a una revisión bibliográfica en las bases de datos de Web of Science, Scopus y PubMed, con información de los últimos diez años. Se encontró que la mencionada terapia favorece la calidad de vida emocional del paciente.

ABSTRACT. Depression in cancer patients is considered a prevalent psychological problem since painful symptoms increase when indicated as a chronic disease; in some cases, activities are excepted, becoming an obstacle for the patient in his performance in an integral way. There are various psychotherapeutic techniques as adjuncts to pharmacological treatment to control depression. Therefore, this study aims to analyze the efficacy of behavioral activation therapy in cancer patients with depression problems. A qualitative approach was used based on a bibliographic review in Web of Science, Scopus, and PubMed, with information from the last ten years. It was found that the therapy above favors the emotional quality of life of the patient.

RESUM0. A depressão em pacientes com câncer é considerada um problema psicológico prevalente, uma vez que os sintomas depressivos aumentam quando é indicada como uma doença crônica, em alguns casos as atividades são excluídas, tornando-se um obstáculo para o paciente em sua atuação de forma integral. Existem várias técnicas psicoterapêuticas como coadjuvantes ao tratamento farmacológico para o controle da depressão, portanto, este estudo tem como objetivo

\footnotetext{
${ }^{1}$ Correspondencia: karinaperez301097@gmail.com
} analisar a eficácia da terapia de ativação comportamental em pacientes oncológicos com problemas depressivos. Utilizou-se uma abordagem qualitativa a partir de revisão bibliográfica nas bases de 


\section{INTRODUCCIÓN}

Dimidjam et al. (2011) refieren al cáncer como una enfermedad crónica que afecta la estabilidad, tanto psicológica como física de los sujetos que lo padecen, también son afectados los miembros de la familia que dan origen a una serie de sentimientos negativos. La depresión es un problema que afecta a la salud mental y está a su vez va en aumento, la prevalencia de este trastorno oscila entre el 4 y 5\% a nivel global, además se encuentra asociada a niveles altos de recaída y recurrencia en el trayecto de vida. Para la presentación clínica en la depresión se utilizan criterios de diagnósticos del Manual Diagnóstico y Estadístico de los Trastornos Mentales o DMSV (en inglés Diagnostic and Statistical Manual of Mental Disorders). Permite definir en adultos, adolescentes y niños, por ejemplo: la posibilidad de un estado anímico irritable como síntoma principal ya sea en niños o adolescentes se implica a distintas etapas vitales de forma independiente de la edad.

Es común evidenciar trastornos depresivos en los pacientes oncológicos, la tristeza, el duelo, la ira entre otros sentimientos. Sin embargo, cuando estos se agudizan es un motivo de preocupación ya que la depresión es una enfermedad grave que requiere tratamiento inmediato y con eficacia. Por lo tanto, para tratar la depresión se puede incluir tratamiento farmacológico, psicoterapéutico o combinado con el objetivo de reducir síntomas emocionales negativos favoreciendo a una mejor calidad de vida en el paciente (Aldas, 2015).

La activación conductual (AC) es una terapia psicoterapéutica que motiva a la persona a retomar o emprender actividades que sean importantes y significativas. La misma consiste en planificar acciones, observar las conductas y analizar situaciones concretas que requieren modificación en el comportamiento. Cabe señalar que, al aplicar la terapia, los pacientes depresivos han demostrado alta eficacia de recuperación, pues la amplia evidencia científica en el ámbito empírico fortalece su valor (Uphoff \& Ekers, 2010).

La AC tiene validez empírica en el tratamiento no farmacológico para la depresión debido a su gran efectividad. Muestra superioridad en relación a otras terapias derivadas de la TCC (Terapia Cognitiva Conductual) con efectos similares o superiores a los antidepresivos. Es una terapia flexible y se centra directamente en las necesidades que presenta el paciente esto debido a su orientación hacia la variación del patrón de evitación experiencial y la promoción de la terapia (Dimidjam et al., 2011).

Por tal razón, es fundamental conocer los efectos, componentes y los procesos psicoterapéuticos de la AC en los pacientes oncológicos que presentan cuadros depresivos, la revisión de estudios previos sobre esta terapia para el control de la enfermedad permitirá conocer la utilidad de la técnica, para establecer una guía terapéutica 
para los profesionales de la salud psicológica. El objetivo principal de la presente investigación es analizar la eficacia de la terapia AC (Activación Conductual) en pacientes oncológicos con problemas depresivos.

\section{MÉTODO Y MATERIALES}

Como criterio de elegibilidad, considerando el objetivo de estudio de esta revisión, se buscó identificar todos los artículos que habían realizado explícitamente una intervención centrada en AC (Terapia de Activación Conductual) en pacientes oncológicos con depresión, independientemente del diseño del estudio, al emplear grupos de comparación, o los detalles de la dificultad psicológica. Los términos utilizados para la investigación fueron: terapia de activación conductual, depresión y pacientes oncológicos.

En las bases de información, se realizaron búsquedas en Web of Science, Scopus y PubMed. Los estudios deberán tener información desde 10 años atrás en idioma inglés o español. La búsqueda se completó hasta el 03 de agosto del 2021, en la cual, también se buscaron artículos potencialmente relevantes en las referencias incluidas en los estudios.

\section{RESULTADOS Y DISCUSIONES}

La terapia de AC es la que afronta a los problemas depresivos, pues se dice que es el que interacciona con las diversas conductas que se da en el contexto (Álvarez \& Paredes, 2018). El efecto no se basa de principio genético o intrapsíquico, pues evalúa patrones personales de vinculación con el ambiente para dar una explicación primordial, a fin de que exista cambios a través de la interacción para introducirse a fuentes nuevas o variadas y de esta forma evitar conductas retrogradas.

Las investigaciones iniciales de AC (Activación Conductual) con adolescentes con cáncer resultaron alentadores (Genise, 2014) Se llevó a cabo un estudio que determine la eficiencia de la terapia para que en la franja etaria se implemente el programa denominado Adolescent - Behavioral Activation Program (A - BAP). Se realizó un ensayo aleatorio y controlado con 60 adolescentes clínicamente referidos con un trastorno depresivo, recibiendo 14 sesiones del programa mencionado. La muestra se componía de $64 \%$ mujeres y $36 \%$ varones. La edad promedio fue de 14.9 años. A partir de este estudio se observa que el modelado de efectos mixtos lineales por intención de tratar y el análisis de regresión revelaron que ambas afecciones produjeron una mejora estadísticamente significativa en el trascurso del tratamiento para la depresión, el funcionamiento global, la activación y evitación.

Por lo tanto, mencionada terapia corresponde a un procedimiento psicoterapéutico ampliamente estructurado con carácter ideográfico y metodología flexible hacia las necesidades que presentan los pacientes. Su objetivo 
principal radica en conseguir que los pacientes con cuadros depresivos puedan desarrollar múltiples formas para generar el orden de sus vidas y su ambiente. Se busca que haya un restablecimiento del contacto a través de fuentes de un valor significativo, gracias a su enfoque funcional, el planteamiento contextual y las técnicas que contiene esta terapia es considerada parte del corriente cognitivo conductual (Barraca, 2010).

Gaynor et al., (2015) implementaron un programa de AC en 12 pacientes clínicos quienes habían sido detectados con cáncer, presentaban sintomatología moderada de depresión. El tratamiento consistió 1 sesión de 1 hora de duración por 12 semanas. Los autores refieren mejorías de 12 pacientes estudiados. Cabe mencionar que, durante el tratamiento existió significativamente una disminución de la sintomatología depresiva. Diversos estudios han considerado adaptar el índice psicológico en la sintomatología oncológica para mantener un bienestar emocional al padecimiento oncológico del paciente, de esta forma se garantice una integración en el aspecto emocional en distintas etapas y adaptándose a dicha enfermedad así se evite preocupaciones que hablen sobre la situación. Es permite que las respuestas emocionales desde el aspecto individual, social y conductual sean llevadas de una manera muy afable (Cabrera et al., 2017).

En este mismo sentido esta terapia es utilizada por los profesionales de psicología, para tratar a personas depresivas, siendo considerada como propia. No obstante, el transcurso evolutivo de esta terapia ha logrado dividirse en dos formas de tratamiento efectivas pero un poco diferentes: la AC (Activación Conductual) y la (TACD) Terapia de Activación Conductual, la teoría y epistemología en realidad son pequeñas, sin embargo, el énfasis que se atribuyen algunos componentes si difieren de manera razonable. Ambas son incorporadas en los manuales más reconocidos para el tratamiento psicológico (Barraca, 2009).

En el artículo de Becerra et al. (2017) han considerado a la AC como una técnica necesaria para tratar pacientes oncológicos depresivos focalizando a incrementar conductas que le vinculen a un ambiente en donde le proporcionen eventualidades con índices de mejorar el estado anímico para obtener una calidad de vida. La AC es considerada como una forma empírica, cuyo resultado muestra flexibilidad y está centrado en necesidades únicas de bienestar en el cual se modifican componentes de evitación experiencial. Cabe señalar que esta terapia aplicada a pacientes oncológicos tiene un propósito de valor significativo para generar comportamientos guiados en los pacientes oncológicos, manifiesta un apoyo social, emocional y todas las prioridades que necesitara. Siguiendo el mismo esquema, en el artículo de Villanueva et al. (2020) plantean el objetivo de adaptación escalar de AC en pacientes oncológicos, al correlacionarlo con lo depresivo, dicha escala dio un resultado significativo dando validez a este constructo.

Cabe recalcar, que la AC posee una extensa evidencia científica que afirma su eficacia en el tratamiento psicológico ya que cuentan con reconocidos trabajos experimentales bien controlados. El objetivo terapéutico 
central está dirigido principalmente hacia el reforzamiento negativo es decir la evitación de paciente hacia determinadas situaciones. Por lo contrario, en la TACD el foco de atención del psicoterapeuta se centra en el reforzamiento positivo que se puede obtener del reconocimiento del paciente así mismo como deprimido. De esta manera estas dos ramificaciones, consideran al paciente como un sujeto preso de una situación vital y no un enfermo.

En este sentido al valorar la eficiencia de la AC en pacientes del área oncológica de pulmón y de mama durante su tratamiento oncológico presentan objetivos para reestablecer las actividades cotidianas, incremento de actividades gratificantes, eliminación de enfermedades adheridas e inclusive modificar el patrón de evitación experiencial (Karla, 2017). Los resultados obtenidos en pacientes de cáncer pulmonar al aplicar la terapia AC demostraron cambios significativos a largo plazo dando al grupo una calidad de vida eficiente. Con resultados similares en el grupo oncológico de mama la funcionalidad ha favorecido a un estado físico y emocional satisfactorio, siendo la única molestia las ocasionadas por la intervención quirúrgica.

Dentro de la intervención de AC se considera de vital importancia incorporar dentro de sus intervenciones psicoterapéuticas la motivación del paciente y su compromiso con el proceso psicoterapéutico. Es decir, el paciente se encuentre cómodo con el enfoque presentando para trabajar con sus dificultades, comprendiendo de esta manera los objetivos el estímulo motivador proviene del exterior y no desde adentro del paciente (Martell, 2010). Por consiguiente, se emplean técnicas eficaces y fáciles de aplicar en la modificación conductual, entre ellas tenemos: (1) la programación y estructuración de actividades, (2) solución del problema que interfiere, (3) el reforzamiento positivo directo, (4) el desvanecimiento, (5) el entrenamiento en habilidades sociales, (6) métodos que vinculen de forma directa con la experiencia.

Al tratar de conocer la AC aplicada en pacientes oncológicos hospitalizados desde las creencias y emociones, en el servicio del Hospital Juárez de México Becerra et al. (2013) diseñan un programa de intervención psicológica basadas en la terapia $\mathrm{AC}$, con redes semánticas. Estas favorecen a un constructo de tres estímulos tanto en la estancia hospitalaria, como me encuentro al estar ingresado y que pensamientos surgen al momento de mantener una hospitalización; se ha denotado en los resultados ser eficaz para el tratamiento depresivo, Además, en un análisis conductual se evidencia ventajas y desventajas que se dan posterior al tratamiento. Las tres hipótesis planteadas al paciente ayudan a relacionar habilidades del proceso e irlas rediseñando para que pueda ejercer modelos productivos.

Las técnicas utilizadas en la terapia de AC se basan en aportaciones científicas que parten de la evolución, las cuales proponen un esquema de intervención que contenga la fase de evaluación, en donde se genera la alianza terapéutica tras los contactos iniciales y la recolección de manifestaciones depresivas, se establecen horarios 
de intervención con el paciente y la organización en el tiempo (Barraca , 2016). El terapeuta indica la importancia de la automonitoreo del paciente en sus registros diarios y de comprobar el reforzador asociado. La utilización de distintos cuestionarios apoyará al terapeuta en la fase de evaluación y el desarrollo del tratamiento.

La AC cuando se valora su funcionalidad en el afrontamiento del cáncer con objetivos concretos de intervención evalúan un cambio pre y post seguimiento dan como resultado una recuperación de actividades particulares y domésticas que disminuyen patrones evitacionales para conseguir que las conductas sean reducidas y se mantenga una mejoría positiva (Villoria et al., 2020) . Colaboran así con el control de la enfermedad. Es preciso enfatizar, que la AC no está dirigida al acceso ni a una base placentera que induzcan a actividades de mejoramiento anímico, pues, se direcciona al incremento de actividades que la paciente acceda de forma recompensatoria o ayude a vislumbrar su poblemática (Bianchi \& Henao, 2015).

Una correcta y profunda evaluación de la situación del paciente permitirá al psicoterapeuta establecer los objetivos que se perseguirán durante y después del tratamiento. Estos deberán estar enfocados en la activación conductual bajo el restablecimiento de las fuentes que proporcionan refuerzos positivos. La fase de incorporación de las técnicas se realiza una vez delimitados los objetivos y como punto pretérmino se realiza la fase de solución de problemas, la misma que se basa en su consecución en las claves generales que ayudaran al tipo de afrontamiento del paciente a los problemas. Bajo esta premisa permitirá al sujeto a lograr comprender las fuentes o canales ambientales de su depresión e identificar las conductas que la mantienen (Barraca , 2016). Un resultado similar se ha encontrado en un estudio donde se utilizó la terapia AC en personas con dolor crónico y enfermedad renal crónica, de esto se evidencia cambios de suma importancia que implican al individuo sobre su actividad diaria antes y después del tratamiento (Lundervold, 2016).

Otras investigaciones llevadas a cabo con otros tipos dentro del área hospitalaria, uno de ellos con pacientes oncológicos, que referían algunos indicadores de depresión después de un contexto de intervención, dieron como resultado mejorías con respecto a los índices de glicemia y en el estado de ánimo (Schneider et al., 2020). Con respecto al contexto de la salud directamente a pacientes con problemas oncológicos han dado resultados positivos al implementar la AC. En un estudio con 6 pacientes hospitalizados con diagnóstico de cáncer y sintomatología depresiva se le aplicó un protocolo de AC, donde se evidencia una reducción de la sintomatología en el periodo de tres meses posterior al tratamiento con un resultado eficiente que avale a la terapia enfocada a la depresión (Hopko D. \&., 2005).

En el estudio contextual-funcional de un paciente oncológico para valorar la calidad de vida, el estado emocional con una intervención de la $\mathrm{AC}$ mediante principios y técnicas que integren un constructo de actividades gratificantes para la calidad de la sintomatología emocional favoreciendo de forma eficiente a la terapia con una 
evolución favorable en el ámbito psicológico, pues la correcta monitorización que se programe en actividades brinda buenos resultados (Fernández et al., 2014).

La mayoría de las investigaciones buscan demostrar la efectividad de la terapia de activación conductual en personas diagnosticadas con depresión, por lo cual, han realizado estudios con diseños experimentales y nomotéticas, lo que ha permitido la elaboración de metaanálisis que muestran su eficacia comparada con otras intervenciones de tipo farmacológica o psicológica. Sin embargo, los resultados aún no son concluyentes, pues algunos de ellos señalan que la terapia Cognitivo Conductual y la Activación Conductual presentan resultados positivos en el tratamiento de la depresión (Mazuchelli, 2019).

En un análisis de estudios de la veracidad de mencionada terapia en el tratamiento de pacientes oncológicos han favorecido el estado emocional y físico. En un grupo experimental se ha conseguido que la persona mantenga actividades relevantes y rutinarias dando un resultado satisfactorio, de manera que se eliminen conductas relacionadas hacia la enfermedad y que modifique el patrón de evitación experiencial. En este caso el terapeuta inducía al paciente a analizar de forma contextual rutinas cotidianas, síntomas, restricciones físicas y emociones que preocupen o pongan en duda tanto a la enfermedad como al tratamiento. De tal forma, que la persona se autoeduque a través de la observación con parámetros que determinen de lo que hace, siente y piense con relación a su entorno. De esta forma pueda identificar eventos que debiliten su comportamiento progresivo (González et al., 2019).

Para determinar que terapia era la más efectiva en casos de depresión, se aplicaron en la Activación Conductual y la terapia Cognitivo Conductual en dos grupos de pacientes con diagnóstico de depresión moderada. Luego de la implementación de las intervenciones en cada uno de los grupos se encontró que la implementación de la terapia Cognitiva Conductual no producía cambios superiores en la disminución del síntoma depresivo. Por otra parte, la Activación Conductual producía en sí misma mejorías de manera más rápida permitiendo al paciente mejorar su calidad de vida (Eldason, 2013).

A fin de trabajar sobre el estado animico del paciente con una intervención psicosocial de estructuración de la AC se ha examinado el impacto y viabilidad que existe en cierta terapia mediante una programación que adopte a sobrevivientes oncológicos de mama por medio de ensayos clínicos. González et al. (2017) definien la AC como un tratamiento de intervención tanto en la sintomatología depresiva y ansiedad, inclusive como se lleva a cabo en el trascurso de los días permitiendo explorar la viabilidad de la AC. Algunos hallazgos dentro de este estudio ratifican el impacto de esta terapia AC como significativa en la reducción de los sintomas depresivos.

La terapia AC es considerada como una alternativa a los modelos que poseen un déficit para el tratamiento de los sintomas depresivos en el ámbito clínico desde los esquemas de química cerebral y mecanismos 
psicológicos. Se ha considerado desde la práctica clínica al uso e inclusive una ventaja para desmedicalizar a esta patologia depresiva, esto debico a que la AC es ideográfica, posee un análisis funcional tanto del comportamiento depresivo como actividades planteadas. La AC propone acciones de relevancia incorporando una aceptación de cambio que propiamente se vincula a la aceptación, de tal forma se consiga la modificación de dependencias "experiencia depresiva", reconociendo la pertenencia a un tratamiento con posibilidades a mantener una conducta abierta (Pérez, 2007).

Una gran ventaja que ha demostrado la intervención de la AC en comparación con otras intervenciones terapéuticas es la preparación que tienen los psicoterapeutas en su aplicación, así como el tiempo de ejecución en la práctica clínica. Se interviene sobre los factores específicos de la patología y a partir de una planificación en consideración de los pacientes dentro de las sesiones. El extenso campo de acción, el impacto, los resultados y posibilidades de desarrollo indican que la $\mathrm{AC}$ es una herramienta muy efectiva en el tratamiento de los trastornos psicológicos frente a otras terapias, lo que ha permitido a esta terapia situarse como una de las estrategias terapéuticas válidas para el tratamiento de la depresión (Hopko \& Enriquez, 2017).

\section{CONCLUSIONES}

En este trabajo de investigación se demostró que la depresión es un problema de salud mental que tiene repercusión individual, social y familiar, los profesionales de la salud podrían dar a los pacientes un tratamiento integral para que tengan una mejor calidad de vida. Sería importante que el terapeuta detecte los síntomas de la depresión en los pacientes oncológicos para que dé un diagnóstico adecuado y ayude al paciente a que se reconozca como un individuo preso de una situación y no un enfermo, el terapeuta y el paciente deberán tener los mismos objetivos para que el paciente afronte sus problemas emocionales.

La terapia de tercera generación con bases científicas permitirá que el paciente modifique su conducta mediante la planificación de actividades, resolución de problemas y reforzamiento positivo, por lo cual es necesario hacer una correcta evaluación y establecer metas para la aplicación del tratamiento en beneficio del enfermo. Por otra parte, la terapia de aceptación y compromiso tiene poca evidencia debido a la falta de muestras y de seguimiento para su utilidad, sería valioso realizar estudios con muestras considerables para que aporte eficacia, futuro estudios empíricos y evaluaciones pre y post daría cuenta de los progresos y modificaciones del descenso de la sintomatología.

Conflicto de intereses / Competing interests:

La autora declara que no incurre en conflictos de intereses.

Rol de los autores / Authors Roles:

No aplica.

Fuentes de financiamiento / Funding: 
La autora declara que no recibió un fondo específico para esta investigación.

\section{Aspectos éticos / legales; Ethics / legals:}

La autora declara no haber incurrido en aspectos antiéticos, ni haber omitido aspectos legales en la realización de la investigación.

\section{REFERENCIAS}

Aldas, L. (2015). Problemas cognitivos o cambios en la conducta deprimente. American Cancer Society, 3(1), 210-220. http://dx.doi.org/896977.44/75123-1210.

Álvarez, M., \& Paredes, J. (2018). Activación Conductual: evidencia contextual y empírica. Avances de la Psicología, 4(7), 300-420. http://dx.doi.org/445678-34.34/77743.

Barraca , J. (2016). La Activación Conductual en la práctica: técnicas, organizacion de la intervención, dificultades y variantes. Analisis y Modificacion de la conducta, 23(3), 15-33. http://dx.doi.org/23456.78/443290.

Becerra Gálvez, A., Lugo González, I., \& Reinoso Erazo, L. (2013). Terapia de activación conductual en pacientes oncológicos:una propuesta de intervención. Psicooncología, 10(1), 163-168. http://dx.doi.org/10.5209/reb-psic.2013.v10.41969.

Becerra Gálvez, A., Reinoso Erazo, Y., Bravo González, M., \& Ordaz Carrillo, M. (2017). Activación conductual para el control de la depresión en pacientes oncológicos: revisión. Psicooncología, 4(14), 2-3. http://dx.doi.org/10.5209/psic.57080.

Bianchi, J., \& Henao, A. (2015). Activación conductual y depresión: Conceptualización, evidencia y aplicación en Iberoamérica. Terapia Psicológica, 33(2), 69-80. http://dx.doi.org/10.4067/s071848082015000200002.

Cabrera Macías, Y., López González, E., \& Arredondo Aldán, B. (2017). La psicología y la oncología: en una unidad imprescindible. Revista Finlay, 2(7), 115-127. http://dx.doi.org/S22221-24342017000200007.

Dimidjam, S., Hollon, R., \& Dobson, K. (2011). feelings and Cancer. National Cancer Institute, 3(11), 70-123. http://dx.doi.org/667789./89-45345678.

Eldason, K. (2013). Activacón Conuctual: efectividad y estudios. Psicothema, 8(10), 200-230. http://dx.doi.org/1234.45/78.893245.

Fernández Rodríguez, C., Villoria Fernández, E., Fernández García, P., \& González Fernández, S. (2014). Efectos de la activación conductual en la calidad de vida y estado emocional en los pacientes con cáncer de pulmón. Psicooncología, 2(3), 199-215. http://dx.doi.org/10.5209/rev-PSIC.2014.v11.n2-3.47383. 
Gaynor, E., Palacios, T., \& Trajano, N. (2015). Activacion conductual en pacientes con cáncer terminal. Psicología y Oncología, 41(5), 75-96. http://dx.doi.org/326514-98.78941.

Genise, G. (2014). Implementación de la Activación Conductual para la depresión en la adolescencia: una revisión sistemática de la literatura. Calidad de Vida y Salud, 8(13), 30-41. http://dx.doi.org/56789432.98765/4321.

González Fernández , S., Fernández Rodríguez, C., Padierna Sánchez, C., Besteiro González, J., \& Pérez Álvarez, M. (2019). Activación conductual en cáncer: revisión de tratamientos y evidencias. Revista Argentina de Clínica Psicológica, XXVIII(2), 140-153. http://dx.doi.org/10.24205/03276716.2018.1087.

Hopko, D. \&. (2005). Behavior Therapy for Depressed Cancer Patients in Primary Care. Psychotherapy: Theory, Research, Practice, Training, 42(5), 236-243. http://dx.doi.org/10.1037/0033-3204.42.2.236.

Hopko, Y., \& Enriquez, Z. (2017). Pacientes oncológicos con problemas de depresión. Psicothema, 14(9), 2446. http://dx.doi.org/12345.76/549098765.

Karla, C. (2017). Eficacia de la Activación Conductual en pacientes con cáncer. Psicoonlogía y psicoterapia, 24(3), 1009-1026. http://dx.doi.org/23456.87-333.7898756.

Lundervold, G. (2016). Activación Conductual: un estudio con pacientes con enfermedades crónicas. Psicooncología, 6(32), 23-57. http://dx.doi.org/221345.76/890754332.

Martell, C. (2010). Behavioral Activation for Depression: A. Revista de Psicoterapia, 9(3), 324-540. http://dx.doi.org/10.1111/cpsp.1214-032.

Mazuchelli, L. (2019). Terapia Cognitivo Conductual y Activación Conductual en la depresión. Psicoterapia, 13(5), 234-256. http://dx.doi.org/33324,456/90876.

Pérez, M. (2007). La activación conductual y la desmedicalización de la depresión. Papeles del psicólogo, 4(3), 97-110. http://dx.doi.org/77828208-44/78.

Schneider, P., López, F., \& Rodríguez, D. (2020). Activación Conductual como tratamiento. Revista de Psicoterapia, 10(2), 210-249. http://dx.doi.org/232456/7.90876.

Uphoff, H., \& Ekers, D. (2010). Activación Conductual para la depresión en adultos. Cochrane, 13(3), 32-45. http://dx.doi.org/22367.7/43908765. 
Villanueva Torres, Y., Jurado Cárdenas, S., \& Ramírez Ramírez, A. (2020). Adaptación de la escala de Activación Conductual para la depresión (BATS) en pacientes con cáncer. Psicooncología, 1(37), 25-39. http://dx.doi.org/10.5209/psic.68239.

Villoria, E., Fernandez, C., Fernández, P., \& Peláez, I. (2020). La activación conductual (AC) en mujeres con cáncer de mama durante la quimioterapia: análsis de la evolución de casos. Psicooncología, 1(17), 11 24. http://dx.doi.org/10.5209/psic.68238. 